\title{
Nonequilibrium phase transitions in stochastic systems with coloured fluctuations
}

\author{
D.O.Kharchenko, A.I.Olemskoi, I.A.Knyaz' \\ Sumy State University, Ukraine
}

Received July 12, 2005

\begin{abstract}
We study the behaviour of a class of stochastic spatially extended systems exhibiting transition to absorbing configurations, reentrant noise induced phase transitions and phase transitions induced by noise crosscorrelations. We discuss the behaviour of the system in the presence of multiplicative fluctuations: a possibility of escaping from the absorbing state and the nature of disordered phase appearing beyond the second critical point of the reentrant phase transition. Making use of the mean field approach we have shown that noise cross-correlations lead to continuous, discontinuous and reentrant phase transitions.
\end{abstract}

Key words: noise, absorbing state, order parameter

PACS: $05.10 . \mathrm{Gg}, 05.40 . \mathrm{Ca}, 05.70 . \mathrm{Fh}$

\section{Introduction}

For the last three decades we have observed a considerable increase in the research of nonequilibrium phenomena in macroscopic systems for the purpose of explaining the constructive role of fluctuations of the environment in which the system is placed. An incomplete list of such processes includes noise induced unimodal-bimodal transitions [1], noise induced spatial patterns and phase transitions [2], transition to an absorbing configuration where the system can get trapped [3], etc. In general case, considering the stochastic dynamics, one should deal with a problem of taking into account the correlations between random sources. Several special methods have been developed to this end. Most popular of them are as follows: (i) the cumulant expansion method [5,4]; (ii) the spectral width expansion method $[1,6]$; (iii) the unified colored noise approximation $[7,8]$.

It appears that when the system is nonlinear, the spatial coupling and noise correlations force the system to exhibit a special behavior known as reentrant phase transition [8,9]. It was shown that the reentrant phase transition is observed if the system is perturbed by multiplicative noise (caused by the effect of the environment). A special type of behaviour of stochastic systems is observed in the case of several noise effects with cross-correlations leading to a remarkable and counterintuitive phenomena related to the transformation of phase transition type $[10,11]$.

Despite the colorful picture of the ordering role of fluctuations there are open questions concerning the behavior of the extended system in the presence of different types of noises. First we examine the possibility of the system exhibiting an absorbing configuration to escape from this state due to the spatial coupling. Next we consider the reentrant phase transition induced by the multiplicative noise and discuss the system states in different domains of the values of the control parameter. Finally, it will be shown that due to temporal correlations of the fluctuating sources one can define a discontinuous behavior of an order parameter. To this end we explore an extended stochastic system that obeys the archetypical model of Brownian particle. Within the simplest model with drift caused by Landau-like potential and two colored (multiplicative and additive) noises, we illustrate that phase transitions of both continuous and discontinuous character are realized as biased phase transitions. 


\section{Model and assumptions}

Let us consider an extended system with many microscopic variables. A typical example is a periodic array of atoms (labeled by index $\alpha$ to numerate the site of the array) described by spin variable $s_{\alpha}$, we assume the sites to be separated by the interatomic distance $a$. Next, dividing the system into mesoscopic cells with lateral size $\ell$, where $a \ll \ell \ll L, L$ is a macroscopic length of the system and introducing $N_{i}$ as the number of atoms belonging to the cell $i$, one can define the field variable as the spatial average of the density of the microscopic degrees of freedom: $x_{i}=\left(\ell^{d} N_{i}\right)^{-1} \sum_{\alpha \in i} s_{\alpha}$, here $d$ is a spatial dimension. In a continuous limit we pass to the field variable $x(\mathbf{r}, t)$, with $\mathbf{r}$ being a $d$-dimensional vector denoting the position in space. An evolution of $x \equiv x(\mathbf{r}, t)$ obeys the Langevin dynamics which without loss of generality is determined by the stochastic differential equation in the form

$$
m \partial_{t t}^{2} x+\gamma \partial_{t} x=-\frac{\delta \mathcal{F}[x]}{\delta x}+g_{\mu}(x) \zeta_{\mu}(\mathbf{r}, t)
$$

Here $m$ is the mass parameter, $\gamma$ is a kinetic coefficient, the functional $\mathcal{F}[x]$ plays the role of the free energy potential being in the Ginzburg-Landau form

$$
\mathcal{F}=\int\left[V(x)+\frac{D}{4 d}\left|\partial_{\mathbf{r}} x\right|^{2}\right] \mathrm{d} \mathbf{r}
$$

with $V(x)$ and $D>0$ being a specific thermodynamic potential and an inhomogeneity constant. The last term in equation (1), where index $\mu$ numerates different noises to be summarized in accordance with the Einstein rule, represents Langevin forces which act with amplitudes $g_{\mu}(x)$ and stochastically alternating functions $\zeta_{\mu}$. Neglecting space correlations, we focus on time correlations between forces $\zeta_{\mu}$, i.e.,

$$
\left\langle\zeta_{\mu}(\mathbf{r}, t) \zeta_{\nu}\left(\mathbf{r}^{\prime}, t^{\prime}\right)\right\rangle=\delta\left(\mathbf{r}-\mathbf{r}^{\prime}\right) C_{\mu \nu}\left(t-t^{\prime}\right)
$$

supposing $\left\langle\zeta_{\mu}(\mathbf{r}, t)\right\rangle=0$.

To introduce a class of stochastic systems with universal behaviour we consider in detail the simplest model of correlated additive and multiplicative noises with amplitudes

$$
g_{a}(x)=1, \quad g_{m}(x)=\operatorname{sign}(x)|x|^{a},
$$

where the exponent is $a \in[0,1]$ and the sign function is introduced in order to take into account the direction of the Langevin force. We shall focus on the prototype system concerning the Landau model with the potential

$$
V(x)=-\frac{\varepsilon}{2} x^{2}+\frac{1}{4} x^{4},
$$

where $\varepsilon$ is a parameter corresponding to the dimensionless temperature counted off a critical value in negative direction. We suppose the noises to be Gaussian distributed with zero mean, white in space and colored in time according to the correlation matrix

$$
\widehat{\mathbf{C}}(\tau)=\left(\begin{array}{cc}
\frac{\sigma_{a}^{2}}{\tau_{a}} \mathrm{e}^{-|\tau| / \tau_{a}} & \frac{\sigma_{a} \sigma_{m}}{\tau_{\mathrm{c}}} \mathrm{e}^{-|\tau| / \tau_{\mathrm{c}}} \\
\frac{\sigma_{m} \sigma_{a}}{\tau_{\mathrm{c}}} \mathrm{e}^{-|\tau| / \tau_{\mathrm{c}}} & \frac{\sigma_{m}^{2}}{\tau_{m}} \mathrm{e}^{-|\tau| / \tau_{m}}
\end{array}\right),
$$

where $\sigma_{a}$ and $\sigma_{m}$ are amplitudes of additive and multiplicative noises respectively, $\tau_{a}$ and $\tau_{m}$ are the corresponding autocorrelation times, $\tau_{\mathrm{c}}$ is the time of cross-correlation between the noises. The above autocorrelation functions assume noise terms to be presented with the help of the Ornstein-Uhlenbeck process:

$$
\tau_{\mu} \frac{\mathrm{d} \zeta_{\mu}}{\mathrm{d} t}=-\zeta_{\mu}+\xi_{\mu}(t), \quad\left\langle\xi_{\mu}(t)\right\rangle=0, \quad\left\langle\xi_{\mu}(t) \xi_{\nu}\left(t^{\prime}\right)\right\rangle=\delta_{\mu, \nu} \delta\left(t-t^{\prime}\right)
$$

In the case $D=0$ one can see that in our model three well-known cases arise naturally. Indeed, at $a=0$ we pass to the thermodynamical system with additive fluctuations, at $a=1 / 2$, our model addresses the directed percolation processes and at $a=1$ we arrive at the picture of population dynamics (Maltus-Verchulst model). Other choices of the exponent $a$ allow us to generalize the system and investigate a whole class of models. 


\section{Limit of one multiplicative noise}

As was shown in [2] spatially distributed stochastic systems with coloured noise undergo reentrant noise induced phase transitions where the system states acquire the properties of thermodynamic phases or passes to the absorbing configuration [3]. Below we will present that due to the combined effect of spatial coupling and nonlinearity of the model the system can escape from the absorbing configuration where the stochastic intermittency is realized and then undergoes reentrant phase transition. Moreover we shall show that a disordered phase appearing beyond the second critical point of the reentrant phase transition is of a cluster structure, where distribution over cluster sizes obeys the a power-law form.

If $D=0$ at $a<1 / 2$ and $\varepsilon>0$, then the system passes to the minima of the bare potential $V(x)$. The stationary distribution of the system states has a power-law form $P_{0}(x) \propto x^{-2 a}$ in the limit $x \ll 1$ at $a<1 / 2$. If $a>1 / 2$ the multiplicative noise makes of $x=0$ an absorbing state where the system can get trapped: neither fluctuations nor the deterministic dynamics can push the system away from this configuration. This is because fluctuations disappear at $x=0$, i.e. $g(0)=0$. It provides that the whole system is concentrated in the state $x=0$ where the stationary distribution is $P_{0}(x) \sim \delta(x)$. One can mean that the absorbing configuration formation leads to the ergodicity breaking where only one state $x=0$ is allowed, while others are forbidden.

It is known that due to spatial coupling $(D \neq 0)$ the nonlinear stochastic system can escape from the absorbing state $x=0$, moving into an active one $x \neq 0$ [12]. To see this effect in our system we first consider the white noise limit, supposing $\left\langle\zeta_{m}(t) \zeta_{m}\left(t^{\prime}\right)\right\rangle=\delta\left(t-t^{\prime}\right)$. If the spatial coupling is introduced $(D \neq 0)$, then diffusion and noise play opposite roles. While noise develops the regions of the absorbing state, diffusion favors the dominance of $x$ values fluctuating around minima of $V(x)$. Space-time evolution of the system with $D \neq 0$ at $a<1 / 2$ and $a>1 / 2$ is shown in figure 1 respectively. It is seen that at $a<1 / 2$ the field $x$ fluctuates in two stable phases with

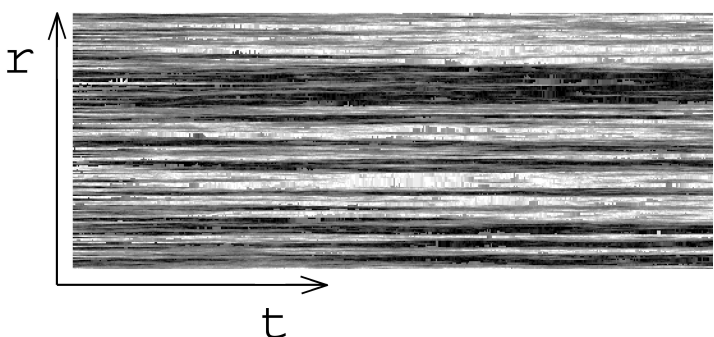

(a)

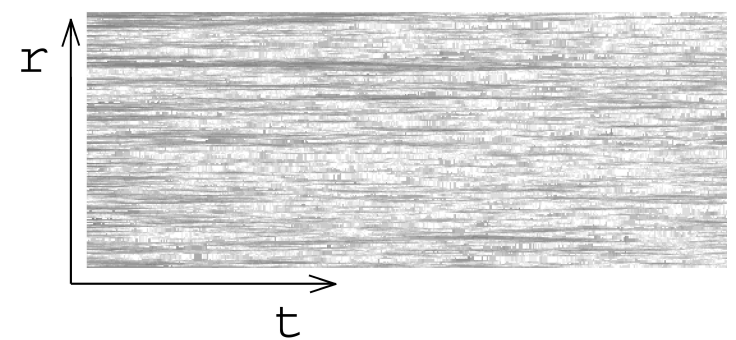

(b)

Figure 1. Space-time evolution of $x(r, t)$ in one dimension at $\varepsilon=1.0$ starting from a random configuration $x \in[-1,1]$ : a) a $=0.3, \mathrm{D}=2.0 ; \mathrm{b}) \mathrm{a}=0.8, \mathrm{D}=2.0$. White, gray and black sites correspond to $x>0, x=0$ and $x<0$ respectively. The time interval is $t \in(0,200)$.

$x<0$ and $x>0$. At $a>1 / 2$ the spatio-temporal intermittency is clearly observed: here a laminar $(x=0)$ phase alternates a turbulent $(x>0)$ phase. The wide range of sizes and life-times of laminar domains are the characteristics of spatio-temporal intermittency; with an increase in $D$ the system moves away from the absorbing state into the turbulent one. Therefore, the diffusion destroys the absorbing configuration and the system passes to the active state. To investigate the role of the exponent $a$ which governs the noise intensity we plot the average spatial fraction $R$ of the system lying in the absorbing phase as an order parameter versus the exponent $a$ in figure 2 . If $R \simeq 0$ the system is in turbulent phase, at $R \simeq 1$ one moves into the absorbing configuration. From figure 2 it is seen that an increase in the noise exponent $a$ at $D \neq 0$ reduces the fraction of the absorbing phase. Analytically one can find that in the uncoupled limit $(D=0)$ at $a>1 / 2$ the stationary distribution is characterized by asymptotic $P_{0}(x) \sim \delta(x)$ which gives $\langle x\rangle=0$. Hence, at $a>1 / 2$ the system is in the disordered (absorbing) state. On the other hand, the limit $D \rightarrow \infty$ can be treated using the saddle-point expansion which yields $\langle x\rangle \neq 0$ [13]. Solutions of this equation formally coincide with the steady-state results of the deterministic analysis for a spatially averaged 


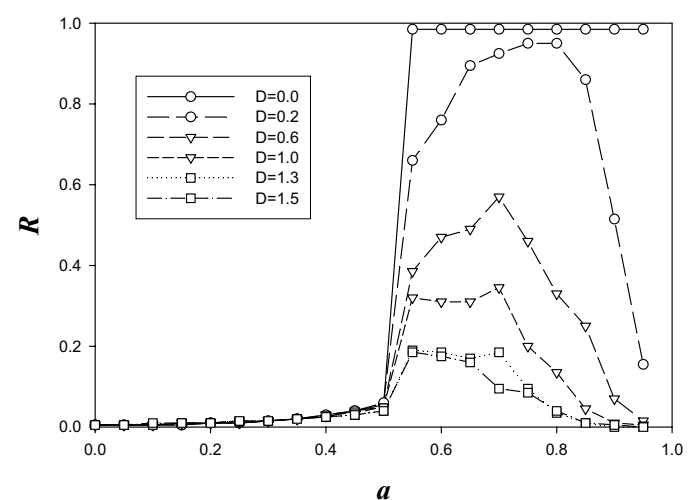

Figure 2. Fraction of the absorbing configuration $R$ vs. the noise exponent $a$ at different values of the diffusion coefficient $D$ at $\varepsilon=1$.

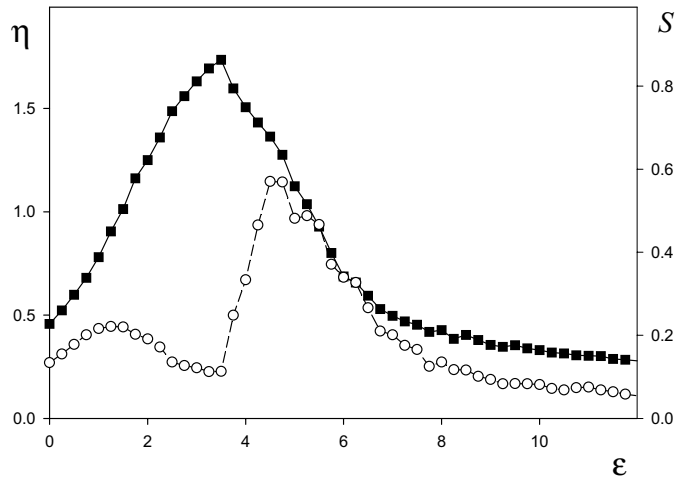

Figure 3. Order parameter $\eta$ (squares) and structure function $S$ (circles) vs. control parameter $\varepsilon$ at $a=0.3, D=1.0, \tau=0.2$.

field, where the transition from the state $x=0$ to the state $x \neq 0$ is realized. According to the obtained picture, we conclude that diffusion is in competition with stochastic component of the evolution of the quantity $x$. Therefore, the spatial interactions provide an escape of the system from the absorbing configuration. Next we focus on the effect of coloured fluctuations in order to investigate the system behaviour in the course of reentrant noise induced phase transition. Despite that, such transitions are characterized by two critical points (above the lower of which the system is passed into the ordered state which exists until the second critical point is achieved), beyond the second critical point the system is determined to be in the disordered state. Introducing the amplitude of the multiplicative noise through the modulus, we assume that the stochastic field $x(\mathbf{r}, t)$ can take values on the entire real axis, i.e $x \in(-\infty, \infty)$ even at $a \in[0,1]$. Our consideration is based on the computer simulations performed for the system with $d=2$ dimension and periodical boundary conditions. In order to find the critical points for the control parameter $\varepsilon$ we investigate the behavior of both an order parameter $\eta$ and a structure function $S$ defined as follows:

$$
\eta=\left\langle\overline{\left|N^{-1} \sum_{i=1}^{N} x_{i}\right|}\right\rangle, \quad S=\left\langle\overline{N^{-1} \sum_{i=1}^{N} x_{i}^{2}-\left(N^{-1} \sum_{i=1}^{N} x_{i}\right)^{2}}\right\rangle,
$$

where the overline corresponds to the averaging over time and angle brackets mean the averaging over the ensemble.

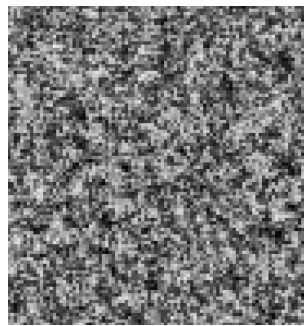

(a)

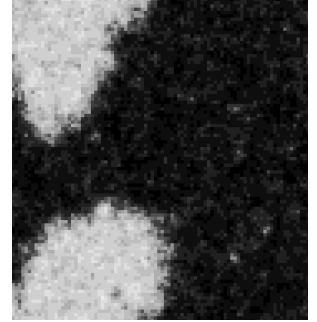

(b)

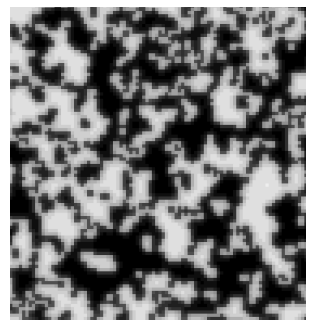

(c)

Figure 4. Typical structures appeared in the course of reentrant phase transition (computer simulations): a) $\varepsilon<\varepsilon_{c 1}$; b) $\varepsilon_{c 1}<\varepsilon<\varepsilon_{c 2}$; c) $\varepsilon>\varepsilon_{c 2}$.

Corresponding computations give a typical form of dependencies shown in figure 3. Here with an increase in the control parameter $\varepsilon$ the order parameter reaches a maximum and then $\eta$ falls 


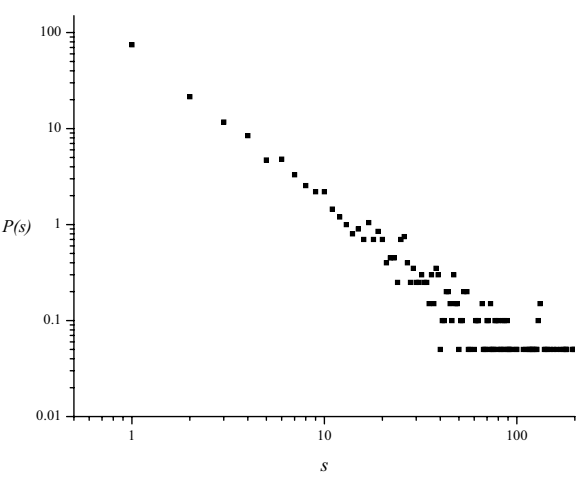

(a)

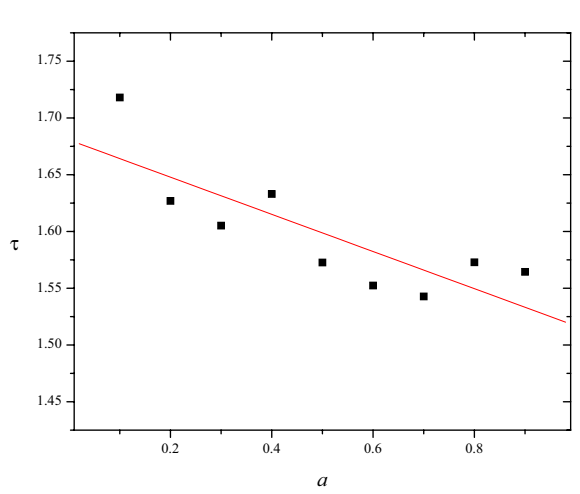

(b)

Figure 5. Power-law dependence of the distribution over cluster size above the second critical point $\varepsilon_{c 2}$ : a) probability density vs. cluster size at $\varepsilon=11.0, D=2, a=0.3$ in double logarithmic plot; b) exponent $\tau$ vs. noise exponent $a$ at $D=2.0, \varepsilon=11.0$.

down. Critical points of such a reentrant phase transition appear in $S(\varepsilon)$ dependence revealing two peaks at $\varepsilon=\varepsilon_{c 1}$ and $\varepsilon=\varepsilon_{c 2}$. A typical form of the structures appearing when the control parameter $\varepsilon$ increases at a stationary limit $(t \rightarrow \infty)$ is shown in figure 4 .

Initially the system is in the disordered state with chaotic configuration (figure 4a) which is out of our interest. In the ordered state $\left(\eta \neq 0\right.$ at $\left.\varepsilon_{c 1}<\varepsilon<\varepsilon_{c 2}\right)$ the system splits into two large domains (figure $4 \mathrm{~b}$ ). The corresponding volume fraction defines the nontrivial magnitude of the order parameter. Beyond the second critical point $\varepsilon>\varepsilon_{c 2}$ (see figure 4c), the order parameter $\eta \rightarrow 0$ and one assumes the transition to the disordered state. However, initial and final disordered states are different. If the former has a chaotic configuration, then the latter has the structure with domains of both negative and positive values of stochastic quantity with different size $s$. Above the second critical point the system state is disordered by means of an equivalence of contributions (positive and negative values of $x$ ) of such domains into an order parameter magnitude. Using the algorithm of marked clusters, we find that the distribution $P(s)$ scales as $P(s) \propto s^{-\tau}$ (figure 5a). As figure $5 \mathrm{~b}$ shows an increase in the index of the multiplicative noise, $a$ decreases the magnitude of $\tau$. It is interesting to note that the magnitude of the index $\tau$ weakly depends on the noise exponent and decreases with an increase in $a$.

\section{Two cross-correlated noises}

In this section, we consider the system with both multiplicative and additive noises being correlated. To make an analytical approach we use the mean field theory where the diffusion is represented through elementary acts of interaction which allows us to make a representation $\Delta x \rightarrow 2 d(\eta-x)$. Here the order parameter $\eta \equiv\langle x\rangle$ is defined through the self-consistency equation

$$
\eta=\int_{-\infty}^{\infty} x P_{\eta}(x) \mathrm{d} x
$$

$P_{\eta}(x)$ is a stationary solution of the corresponding Fokker-Planck equation. The procedure of deriving the effective Fokker-Planck equation is based on the method of kinetic equation for the microscopic probability density $\rho(x, \dot{x}, t)$, which within the framework of cumulant expansion method [5] leads to the evolution equation for the macroscopic probability density function $P(x, \dot{x}, t)=\langle\rho(x, \dot{x}, t)\rangle$, where the averaging is taken over the fluctuations. After integrating over velocities $\dot{x}$, one can write down the evolution equation passing for the distribution $P(x, t)$ in the form

$$
\partial_{t} P=-\partial_{x}\left(\mathcal{D}_{1} P\right)+\partial_{x x}^{2}\left(\mathcal{D}_{2} P\right),
$$


where the effective drift and diffusion coefficients are as follows [11]:

$$
\begin{aligned}
& \mathcal{D}_{1}=\gamma^{-1}\left\{\left(-\partial_{x} V+D(\eta-x)\right)+M_{\mu \nu}^{(1)} g_{\mu} \partial_{x} g_{\nu}\right\}, \\
& \mathcal{D}_{2}=M_{\mu \nu}^{(0)} \gamma^{-2} g_{\mu} g_{\nu} .
\end{aligned}
$$

Here we introduce the moments of correlation functions $M_{\mu \nu}^{(n)}=\int C_{\mu \nu}(t) t^{n} \mathrm{~d} t$. Corresponding calculations give

$$
\begin{aligned}
& \mathcal{D}_{1}=\gamma^{-1}\left[D(\eta-x)+x\left(\varepsilon-x^{2}\right)\right]+a \sigma_{m}|x|^{a-1}\left[\sigma_{a} \tau_{\mathrm{c}}+\sigma_{m} \tau_{m} \operatorname{sign}(x)|x|^{a}\right], \\
& \mathcal{D}_{2}=\gamma^{-2}\left[\sigma_{a}+\sigma_{m} \operatorname{sign}(x)|x|^{a}\right]^{2} .
\end{aligned}
$$

Under stationary condition, the relevant distribution function is of the form

$$
P_{\eta}(x)=\frac{\mathcal{Z}_{\eta}^{-1}}{\mathcal{D}_{2}(x)} \exp \left(\int_{-\infty}^{x} \frac{\mathcal{D}_{1}\left(x^{\prime}, \eta\right)}{\mathcal{D}_{2}\left(x^{\prime}\right)} \mathrm{d} x^{\prime}\right),
$$

where the partition function $\mathcal{Z}_{\eta}$ takes care of the normalization condition. The equation (9) has solutions within the domain bounded by the Newton-Raphson condition

$$
\left.\int_{-\infty}^{\infty} x \frac{\partial}{\partial \eta} P_{\eta}(x)\right|_{\eta=0} \mathrm{~d} x=1
$$

obtained by differentiating equation (9) over the order parameter $\eta$.

We start the consideration of the effect of noise cross-correlations on the system behavior from the solution of the self-consistency equation (9). It is well known in the theory of phase transitions, that the symmetry breaking causes the ordered state corresponding to the solution $\eta \neq 0$, while the disordered phase is related to $\eta=0$. In the absence of the multiplicative noise, the symmetry of the stochastic distribution can be broken only by the spatial coupling which plays the role of a conjugate field related to the order parameter $\eta$. The principal feature of far-off-equilibrium systems with colored noise is that the symmetry can be restored due to the combined effect of both the multiplicative noise and the system nonlinearity $[8,9]$. Therefore, a reentrant phase transition in such systems takes place. Our aim is to demonstrate that the picture of the phase transition can be crucially changed by means of noise cross-correlations.

First, we consider the solution of equation (9) at different values of the noise cross-correlation scale $\tau_{\mathrm{c}}$. As it is shown in figure $6 \mathrm{a}$, in the absence of both noises $\left(\sigma_{a}=\sigma_{m}=0\right)$ and coupling $(D=0)$ the system behaves in an usual manner, as in a square-root law (dashed curve with both vertical derivative in the point of origin and symmetry with respect to the $\varepsilon$-axis). Such a behavior means the appearance of the maxima of the distribution (15) at the points $\pm \sqrt{\varepsilon}$ which can be interpreted as a standard second order transition with the mean value $\eta=0$. The situation changes significantly if we switch on the noises and coupling. First, the above symmetry is broken and only the negative value of the order parameter in the limit of small cross-correlations survives (curve 1). The combined effect of correlated noises, the system nonlinearity and spatial coupling leads to the change of the sign of the order parameter at small values $\eta$. Indeed, as can be seen from the curves 2,3 , an increase in the cross-correlation time $\tau_{\mathrm{c}}$ shifts the negative solution weakly into the positive domain causing the reorientation transition at the driving parameter $\varepsilon_{r}$. In addition to this transition, an increase in the cross-correlation scale leads to the appearance of the positive solutions according to discontinuous phase transitions which are of reentrant nature within the domain bound by both lower $\varepsilon_{\mathrm{c}}$ and upper $\varepsilon^{c}$ boundaries (see elliptic form parts of curves 2, 3 where solid and dotted lines relate to (meta)stable and unstable solutions). With the subsequent growth of the cross-correlation time above the value $\tau_{c r}$ (thin solid curve in figure 6a), the back bifurcation occurs and temperature dependence of the order parameter takes one-connected character. This means that one-link the increase of the control parameter $\varepsilon$ initially leads to the (meta)stable branch of positive magnitudes $\eta$ (solid curve), then the unstable branch (dotted curve) follows 


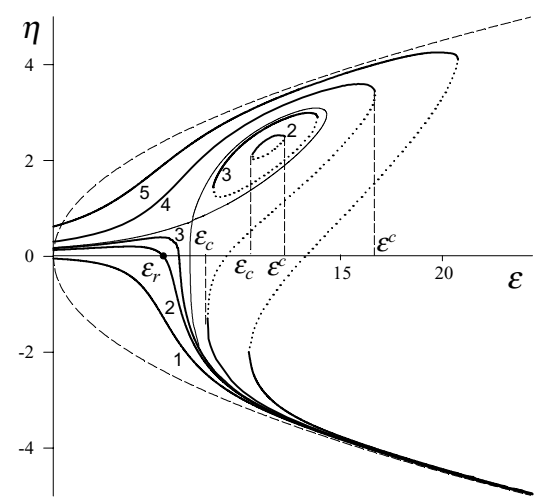

(a)

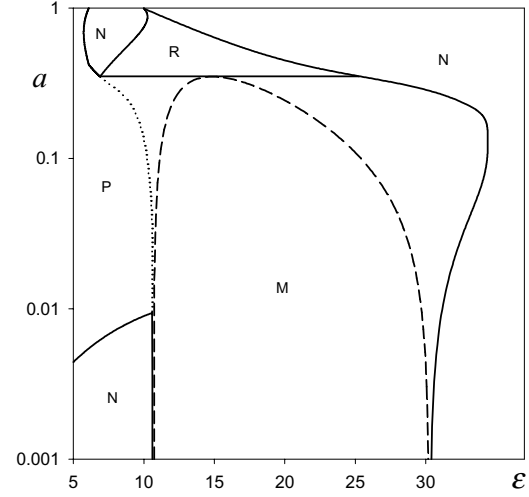

(b)

Figure 6. Stationary behaviour of the system with two correlated noises. a) Dependence of the order parameter $\eta$ on the control parameter $\varepsilon$ at $a=0.8, \sigma_{a}^{2}=4.84, \sigma_{m}^{2}=0.01, \tau_{m}=0.01$, $D=1.0$. Curves 1, 2, 3, 4, 5 correspond to $\tau_{\mathrm{c}} \rightarrow 0, \tau_{\mathrm{c}}=2.5,3.0,5.0,10.0$, respectively. Dashed curve relate to the bare dependence $\eta= \pm \sqrt{\varepsilon}$, dotted curves correspond to the unstable solutions. b) Phase diagram in $(\varepsilon, a)$ plane at $\sigma_{a}^{2}=4.84, \sigma_{m}^{2}=0.01, \tau_{m}=0.01, \tau_{\mathrm{c}}=2.5$, $D=1.0$. Dashed curve relates to the limit $\tau_{\mathrm{c}} \rightarrow 0$.

from the point $\varepsilon^{c}$ down to $\varepsilon_{\mathrm{c}}$ and finally the negative (meta)stable state is merged. A further increase in $\tau_{\mathrm{c}}$ results in the formation of a hysteresis loop in the $\eta(\varepsilon)$ dependence where both (meta)stable and unstable states appear to be the solutions of equation (9) (see curves 4,5 ).

To find the relations between noise exponent $a$ and the control parameter $\varepsilon$ we consider the phase diagram in $(\varepsilon, a)$ plane. It appears that for the noises with weak cross-correlation $\left(\tau_{\mathrm{c}} \rightarrow 0\right)$ the new phase (R-domain) arises only at small enough values of $a$ which define the power of the multiplicative noise (see dashed curve in figure 6b). In other words, considering the class of systems with both additive and multiplicative noises, one should take into account that the ordering processes are possible in the case of weak cross-correlation only if the multiplicative noise has a weak power. For the systems with $a \rightarrow 1$ the weak cross-correlation cannot induce new phase formation. According to the solid-dotted curves in figure $6 \mathrm{~b}$, an increase in $\tau_{\mathrm{c}}$ leads to the appearance of a small valued domain of $\varepsilon$ where the reorientation transition takes place with $a$-growth (domains of positive and negative values of the order parameter are denoted as $\mathrm{P}$ and $\mathrm{N}$ respectively). Besides, domains of both positive and negative order parameters, being reoriented, join with the metastable phase region (M-domain) at small values of the index $a$. However, an increase in $a$ leads to the reentrant phase transition for the long range cross-correlations. At small and moderate values of the noise exponent $a$, the system behavior is described by the hysteresis loop formation.

To understand the main features of the system under consideration we proceed from the effective motion equation for $\eta$ which follows from the corresponding Fokker-Planck equation (10). Within the mean field approach, we get $\dot{\eta}=\left\langle\mathcal{D}_{1}(x)\right\rangle \simeq-\partial_{\eta} F$. Using the definition (13), after self-averaging for a thermodynamic potential we get

$$
F=-\left(\varepsilon \eta^{2}+\tau_{m} \sigma_{m}^{2} \eta^{2 a}\right) / 2+\eta^{4} / 4-\tau_{\mathrm{c}} \sigma_{a} \sigma_{m} \operatorname{sign}(\eta)|\eta|^{a} .
$$

It follows that the multiplicative noise leads to an increase in the control parameter $\varepsilon$ due to the addition of $\tau_{m} \sigma_{m}^{2}$. A smearing of the related dependencies $\eta(\varepsilon)$ at moderate values $\varepsilon$ is induced by the effective field $\tau_{\mathrm{c}} \sigma_{a} \sigma_{m}$ inherent in the cross-correlation effect fixed by the characteristic time $\tau_{\mathrm{c}}$ and intensities $\sigma_{a}^{2}, \sigma_{m}^{2}$.

\section{Conclusions}

We used the generalized model to set up a class of stochastic systems exhibiting a complex behaviour with the formation of the absorbing state, reentrant and biased phase transitions. Con- 
sidering a noise limit we illustrate the possibility of the system to escape from the absorbing configuration and discuss the nature of the disordered state beyond the second critical point of reentrant phase transition. We have shown that noise cross-correlations can induce phase transitions where the order parameter varies discontinuously or in a reentrant manner.

The obtained results can be applied to complex systems which are far-off-equilibrium and hold several collective degrees of freedom. A typical example can be found in solid state physics where a reentrant metastable phase can appear if the matrix phase relates to random ensemble of defects of different dimensions subject to the field of plastic flow (driven dislocation-vacancy ensemble). Here, in the course of plastic flow different defect structures alternate according to the picture of the first order phase transition [14]. The structural reorientation transitions take place where the sign of the order parameter is related to the resulting direction of the Burgers vectors of dislocation cluster.

\title{
References
}

1. Horsthemke W., Lefever R. Noise-Induced Transitions. Berlin, Springer-Verlag, 1984.

2. Garcia-Ojalvo J., Sancho J.M. Noise in Spatially Extended Systems. New York, Springer-Verlag, 1999.

3. Muñoz M.A., Phys. Rev. E, 1998, 57, No. 2, 1377-1383.

4. Shapiro V.E., Phys. Rev. E, 1993, 48, No. 1, 109-120.

5. Van Kampen N.G. Stochastic Processes in Physics and Chemistry. Amsterdam, North-Holland, 1992.

6. Risken H. The Fokker-Planck Equation, Berlin, Springer-Verlag, 1989.

7. Castro F., Wio H.S., Abramson G., Phys. Rev. E, 1995, 52, No. 1, 159-164.

8. Mangioni S.E., Deza R.R., Toral R., et.al.,Phys. Rev. E, 2000, 61, No. 1, 223-232.

9. Van der Broeck C., Phys. Rev. Lett., 1994, 73, No. 25, 3395-3398; Van der Broeck C., Parrondo J.M.R., Toral R., Kawai R., Phys. Rev. E, 1997, 55, No. 4, 4084-4094.

10. Kharchenko D.O., Knyaz' I.A., Eur. Phys. Jour. B, 2003, 32, 375-382.

11. Olemskoi A.I., Kharchenko D.O., Knyaz' I.A., Phys. Rev. E, 2005, 71, 041101(1)-041101(12).

12. Zimmermann M.G., Toral R., Piro O., et. al., Phys. Rev. Lett., 2000, 85, 3612-3615.

13. Gardiner C.W. Handbook of Stochastic Methods. New York, Springer, 1989.

14. Olemskoi A.I., Katsnelson A.A. Synergetics of Condensed Matter. Moscow, URSS, 2003 (in Russian).

\section{Нерівноважні фазові переходи в стохастичних системах 3 кольоровими флуктуаціями}

\author{
Д.О.Харченко, О.І.Олємской, І.О.Князь \\ Сумський державний університет, \\ вул. Римського-Корсакова 2, \\ Суми, 40007, Україна \\ Отримано 12 липня 2005 р.
}

Досліджено клас розподілених стохастичних систем, які проявляють перехід до поглинаючого стану, реверсивні індуковані шумом фазові переходи та фазові переходи, що індуковані шумовими кроскореляціями. Проаналізовано поведінку таких систем за наявності мультиплікативних флуктуацій: можливість виходу із поглинаючого стану та природу неупорядкованої фази, яка виникає за другою критичною точкою реверсивного фазового переходу. В рамках теорії середнього поля, показано, що крос-кореляції флуктуацій приводять до неперервного, переривчастого та реверсивного фазових переходів.

Ключові слова: шум, поглинаючий стан, параметр порядку

PACS: $05.10 . \mathrm{Gg}, 05.40 . \mathrm{Ca}, 05.70 . \mathrm{Fh}$ 